\title{
ISOLATION AND IDENTIFICATION OF SOME INDIGENOUS CYANOPROKARYOTA ISOLATES BASED ON MORPHOLOGICAL AND PHYSIOLOGICAL CHARACTERIZATIONS
}

\author{
Salem, S. H. ${ }^{1}$, Fatma I. EL-Zamik ${ }^{1}$, Howaida M. L. Abd El-Basit ${ }^{1}$, \\ Azza A. Abd El-All ${ }^{2}$ and A. S. Ali ${ }^{1}$ \\ 1- Agric. Microbiology Dept., Fac. Agric., Zagazig Univ., Zagazig, Egypt. \\ 2- Soils Water and Environ. Res. Inst., Agric. Res. Center (ARC), Giza, Egypt.
}

\begin{abstract}
Forty-two cyanoprokaryota strains were isolated from 10 diverse sites along Sharkia Governorate, Egypt 7 sites representing the fertile soils and 3 sites representing the new reclaimed soils). Eight genera and 11 species of $\mathrm{N}_{2}$-fixing cyanoprokaryota were identified based on available morphological distinction and physiological characterization. On the basis of the obtained results, we classified these isolates to 3 genera and 5 species isolated from fertile soils and 7 genera and 8 species isolated from new reclaimed soils. Nostoc spp strains were the most common filamentous heterocyst-forming cyanoprokaryota (22 strains) and two species of Nostoc including 11 strains of $N$. muscorum and 11 strains of $N$. calicicola. Anabaena includes 10 strains in three species (A. oryzae (6 strains), A. circinalis (2 strains) and A. oscillarioides (2 strains). Nodularia spumigena were represented in this work by two strains from fertile soils. In addition Calothrix clavata (2 strains), Synechococcus sp. (3 strains) and one strain of each Oscillatoria homogenea, Dermocarpa olivacea and Xenococcus kerneri were inhabited in the reclaimed soils. This indicates that Nostoc spp. and Anabaena spp. were highly distributed in fertile soils more than new reclaimed soils. The latter species were the most important cyanoprokaryota genera to fix atmospheric nitrogen in rice field.
\end{abstract}

Keywords: Identification, Indigenous Cyanoprokaryota, Morphology, Nitrogenase Activity, Pigment Contents, Physiology.

\section{Introduction}

Cyanoprokaryota are distributed world-wide and diverse assemblage of unicellular or multicellular photosynthetic prokaryotes that possess chlorophyll $a$ and perform oxygenic photosynthesis. Cyanoprokaryota are responsible for a significant proportion of the biological fixation of nitrogen on Earth (Haselkorn and Buikema, 1992). Cyanoprokaryota are major biomass producers both in aquatic as well as terrestrial ecosystems and represent more than $50 \%$ of the biomass in many aquatic ecosystems (Häder $\boldsymbol{e t}$ al., 2007). They are valuable sources of various natural products of medicinal and industrial value (Cardozo $\boldsymbol{e t}$ al., 2007). In addition, their inherent capacity to fix atmospheric nitrogen makes them ecologically important for rice-growing countries where they add to rice fields as natural biofertilizer (Vaishampayan et al., 2001). 
Cyanoprokaryota, as environmentally important microoganisms, has been identified mainly on the basis of morphological characters, such as shape and size of trichomes, cell types, size and location of heterocysts and akinetes, and planes of division (Rippka et al., 1979). Morphological variation of trichomes, heterocytes and akinetes can be used in identification of species of the nostocalean genera (Hindak, 2000).

In the second edition of Bergey's Manual of Systematic Bacteriology (2001) the cyanoprokaryota are subdivided into five quasi-taxonomic groups or subsections. Their diagnosis is based on the dichotomous key: the morphotype is unicellular or quasi-multicellular (trichome); division is binary or multiple (with formation of beocytes); presence or absence of differentiated cells (akinetes and heterocysts); and absence or presence of ramification (true or false) in the trichome (Pinevich, 2008).

The present system of cyanoprokaryota must be modified according to combined markers, in which molecular data (as an indisputable genetic basis) should be correlated with biochemical, ultrastructural, phenotypic and ecological data. New data are necessary in order to correct or up date the system, thus, the classification must continually be revised and supplemented (Komàrek, 2010 a and $\mathbf{b}$ ).

The modern taxonomic system of cyanoprokaryota using morphological, physiological, biochemical and genetic characterizations based upon axenic strains is now generally accepted (Stainer et al., 1971; Rippka et al., 1979; Castenholz and Waterbury, 1989). Therefore, it is necessary to develop the new system of cyanoprokaryota taxonomy from the existing botanical assemblage of names so that compatibility and continuity with the traditional botanical classification system are preserved. This means that morphological characterization and identification at the species level according to botanical concepts remain an initial step (Li et al., 2000).

This study was therefore undertaken to isolate $\mathrm{N}_{2}$-fixing free-living cyanoprokaryota from different rice fields in Sharkia Governorate, Egypt and evaluate their diversity based on their widespread distribution, morphological distinction and physiological characterization, as well as identification of a core set of isolates for further utilization as bioinoculants for rice crop.

\section{Materials and Methods}

\section{Study area and sampling}

Sharkia Governorate is located in the East Delta of North Egypt $\left(30^{\circ} .19 .50\right.$ $\mathrm{N}$ to $31^{\circ} .15 .15 \mathrm{~N}$ ). The total area is $4911 \mathrm{~km}^{2}$ equivalent to $1,169,285$ faddans $\left(\right.$ faddan $\left.=4200 \mathrm{~m}^{2}\right)$. Sharkia is the second governorate at ARE in terms of agriculture planted area (824,098 faddans). It is one of the most important rice growing areas in Egypt (around 176,401 faddans in 2010 season). 
Eighty moistured soil samples were collected from 19 locations cultivated with rice plants situated in 10 different regions at Sharkia Governorate. These regions included seven areas from the fertile soil namely Zagazig $(Z)$, Hihya $(H)$, Mashtool El-Sook (M), Belbiese (B), Diarb Nagm (D), El-Hessenia (E), AboHammad (A), and three areas of the newly reclaimed soils, which suffer from high salinity namely San El-Hagar (Sa), Shader Asaam (Sh) and Sahl Elteen (Se). These locations were chosen to represent wide spread fields of rice crop cultivation in the governorate. The isolation regions are shown in Table (1). The different isolates of cyanoprokaryota were isolated for the aforementioned moistured soil samples.

Table (1) Sites and physicochemical analyses of soils in different locations of Sharkia governorate selected for isolation of cyanoprokaryota.

\begin{tabular}{|c|c|c|c|c|c|c|c|c|c|c|c|c|c|c|c|}
\hline \multirow{3}{*}{$\begin{array}{c}\text { Geographical } \\
\text { sites }\end{array}$} & \multirow{2}{*}{\multicolumn{3}{|c|}{ physical analysis }} & \multirow{2}{*}{\multicolumn{4}{|c|}{ Mechanical analysis }} & \multicolumn{7}{|c|}{ Chemical analysis } & \multirow{3}{*}{$\begin{array}{l}\text { Name of } \\
\text { isolates }\end{array}$} \\
\hline & & & & & & & & \multicolumn{4}{|c|}{ Cations $(\mathrm{mg} / \mathrm{L})$} & \multicolumn{3}{|c|}{$\operatorname{anions}(\mathrm{mg} / \mathrm{L})$} & \\
\hline & pH & $\begin{array}{c}\text { E.C } \\
\text { dsm }^{-1}\end{array}$ & $\begin{array}{c}\text { O } \\
\text { M.\% }\end{array}$ & $\begin{array}{c}\text { Sand } \\
\%\end{array}$ & Silt \% & $\begin{array}{c}\text { Clay } \\
\%\end{array}$ & $\begin{array}{c}\text { Type of } \\
\text { soil }\end{array}$ & $\mathbf{C a}^{++}$ & $\mathrm{Mg}^{++}$ & $\mathbf{N a}^{+}$ & $\mathbf{K}^{+}$ & $\mathrm{HCO}_{3}^{-}$ & $\mathrm{Cl}^{-}$ & $\mathrm{SO}^{-}$ & \\
\hline \multicolumn{16}{|c|}{ Fertile soils } \\
\hline Zagazig (Z) & 7.86 & 2.67 & 1.95 & 33.22 & 13.91 & 52.89 & Clay & 0.55 & 0.60 & 1.65 & 0.07 & 0.28 & 0.81 & 1.70 & \begin{tabular}{|l|} 
Z1,Z.11, \\
Z.12,Z 11
\end{tabular} \\
\hline Hihya $(\mathbf{H})$ & 7.61 & 6.4 & 1.82 & 38.8 & 5.6 & 55.6 & Clay & 10.7 & 11.5 & 43.5 & .54 & 3.0 & 32.6 & 30.6 & \begin{tabular}{|l} 
H.5, H.52, \\
H.13, H.14
\end{tabular} \\
\hline $\begin{array}{c}\text { El-Hessenia } \\
\text { (E) }\end{array}$ & 7.75 & 4.8 & 1.22 & 48.0 & 8.1 & 42.9 & Clay & 6.8 & 6.8 & 34.8 & 0.4 & 3.6 & 8.6 & 3.9 & $\begin{array}{l}\text { E 4, E } 41 \\
\text { E 3, E } 24\end{array}$ \\
\hline $\begin{array}{c}\text { Diyarb Nigm } \\
\text { (D) }\end{array}$ & 7.55 & 17.20 & 1.22 & 30.1 & 15.2 & 54.7 & Clay & 51.5 & 24.0 & 7.5 & 3.2 & 3.6 & 9.6 & 75.7 & $\begin{array}{c}\text { D1, D2, } \\
\text { D13 }\end{array}$ \\
\hline $\begin{array}{c}\text { Abo- } \\
\text { Hammad (A) }\end{array}$ & 7.32 & 1.7 & 1.33 & 35.0 & 31.6 & 33.3 & $\begin{array}{l}\text { Clay } \\
\text { loam }\end{array}$ & 14.8 & 3.3 & 3.01 & 1.9 & 2.2 & 8.1 & 4.2 & A2,A22 \\
\hline Belbeise (B) & 7.40 & 3.50 & 1.22 & 47.2 & 20.8 & 32.0 & $\begin{array}{l}\text { Clay } \\
\text { loam }\end{array}$ & 26.2 & 22.1 & 34.8 & 0.46 & 2.4 & 38.4 & 42.7 & B I, B 4,B 5 \\
\hline $\begin{array}{c}\text { Mashtool El- } \\
\text { Sook }(M)\end{array}$ & 7.10 & 3.10 & 1.08 & 45.0 & 15.8 & 39.2 & $\begin{array}{l}\text { Clay } \\
\text { loam }\end{array}$ & 27.1 & 15.3 & 20.1 & 0.31 & 2.3 & 43.3 & 18.1 & M11, M12 \\
\hline \multicolumn{16}{|c|}{ Newly reclaimed soils } \\
\hline $\begin{array}{c}\text { San El-hagar } \\
\text { (Sa) }\end{array}$ & 7.95 & 19.7 & 1.01 & 34.32 & 12.97 & 52.82 & Clay & 40.0 & 27.8 & 119.6 & 0.50 & 2.7 & 132.4 & 52.8 & \begin{tabular}{|c|} 
Sa 30, Sa \\
31, Sa 32, \\
Sa 33, Sa \\
80, Sa 81, \\
Sa 82, Sa 83 \\
\end{tabular} \\
\hline $\begin{array}{c}\text { Shader } \\
\text { Asaam } \\
\text { (Sh) }\end{array}$ & 8.52 & 21.3 & 0.46 & 42.7 & 13.4 & 53.9 & Clay & 18.5 & 22.6 & 178.0 & 0.44 & 6.8 & 159.0 & 55.1 & \begin{tabular}{|c} 
Sh 1, Sh 10, \\
Sh 11, Sh \\
12
\end{tabular} \\
\hline $\begin{array}{l}\text { Sahl Elteen } \\
\quad \text { (Se) }\end{array}$ & 8.35 & 22.1 & 0.55 & 38.6 & 16.5 & 44.9 & Clay & 17.3 & 21.7 & 196.0 & 0.37 & 10.5 & 168.0 & 56.8 & \begin{tabular}{|c} 
Se12,Se100, \\
Se 80, Se \\
81, Se 82, \\
Se 112, \\
Se 113, Se \\
114 \\
\end{tabular} \\
\hline
\end{tabular}




\section{Isolation and Purification of Cyanoprokaryota}

\section{Development of cyanoprokaryota and culturing conditions}

Each water-soil sample of rice cultivated soil $(10 \mathrm{ml})$ contains about $2 \mathrm{~g}$ of the specific soil was transferred to sterile $250 \mathrm{ml}$ conical flasks containing 90 $\mathrm{ml}$ BG-13 medium. Microscopic observations were recorded, as well as growth appearance, during 3-4 weeks of incubation at $30^{\circ} \mathrm{C}$. Several successive transfers have been made using the respective medium and all the isolated cyanoprokaryota by these enrichment cultures were subjected to a course of purification (Ferris and Hirsch, 1991).

Streaking method (Roger and Kulasooriya, 1980) was carried out using the BG13 agar medium to get single culture from these isolates. The plates incubated at $30^{\circ} \mathrm{C}$ under continuous illumination (400-500 lux). Developed colonies were picked up and microscopically examined and those composed of cyanoprokaryota cells were preserved on slants containing BG 13 medium. All isolates obtained were maintained under photoautotrophic growth conditions in BG 11 medium (Rippka et al., 1979) and BG12 and BG 13 media (Ferris and Hirsch, 1991). Both enrichment and stock cultures were grown under continuous illumination with Philips Florescent 40-W cool-white fluorescent tubes, at a relatively low light intensity between $400-500$ lux and incubated at $30^{\circ} \mathrm{C}$.

\section{Purification of Cyanoprokaryota from heterotrophic bacteria}

Purification of Cyanoprokaryota using antibiotics (nystatin and cycloheximide), was carried out according to Ferris and Hirsch (1991). Also, purification of cyanoprokaryota using UV radiation was employed according to the method described by Garcia-Pichel et al. (1992). Isolates grown in test tubes were subjected to UV radiation by placing the tubes under UV lamp using DESGAUV 254/366 nm, exposed for 30 minutes.

\section{Identification of cyanoprokaryotal Isolates.}

\section{Morphological characteristics revealed by microscopic analysis}

The morphological examination of the selected cyanoprokaryotal isolates was carried out at different stages of growth in N-free BG11 medium. The isolates were viewed under a light microscope, the general shape and color of culture of isolates, shape, width and length of vegetative cells, presence or absence of heterocysts, site of heterocysts in filaments, shape, width and length of heterocysts, presence or absence of akinetes, shape, width and length of akinetes, presence or absence of baeocystes and sheath were described and/or measured using the keys provided by Desikachary (1959), Rippka et al. (1979) and Bergey's Manual of Systematic Bacteriology (2001). 
Analytical procedures for growth attribute pigments and enzymes involved in $\mathrm{N}$-assimilation.

\section{Nitrogenase activity}

The efficient of different isolates to fix atmospheric nitrogen $\left(\mathrm{N}_{2}\right.$-ase activity) was assayed. The enzyme activity was estimated using the acetylene reduction assay technique (ARA) according to the standard procedures by Hardy et al. (1973).

\section{Total nitrogen content}

Total $\mathrm{N}$ content in the cultures was determined by microkjeldahle method (Bremner and Mulvaney, 1982) using $\mathrm{H}_{2} \mathrm{SO}_{4}$ digestion for the cyanobacterial cells followed by steam distillation and estimation of ammonia produced by titration with dilute $\mathrm{HCl}$ and the total protein content was calculated according to Bremner and Mulvaney (1982).

\section{Pigments content}

Chlorophyll $a(\mathrm{Chl} a)$ in the cultures was estimated by the method of Meeks and Castenholz (1971). Amounts of cyanobacterial phycocyanin (C-PC) and cyanobacterial allophycocyanin (C-APC) in each sample were estimated by the method of Bennett and Bogorad (1973) and the modifying procedure by Singh et al. (2009).

\section{Results and Discussion}

Identification of the 42 cyanobacterial isolates based on morphological features such as color of cultures, general shape, width and length of vegetative cells. In addition, presence or absence of heterocysts, shape, width and length of the heterocysts and their site on filaments; presence or absence of akinetes, shape, width and length of the akinetes; presence or absence of baeocystes and sheath were taken into account (Tables 2 and 3). Physiological properties such as total protein, nitrogenase activity, chlorophyll a contents, phycocyanin contents and percentage of chlorophyll $a$ / phycocyanin (Tables 4 and 5) were included. Based on the aforementioned morphological and physiological characterizations, and according to Desikachary (1959), Rippka et al. (1979) and Bergey's Manual of Systematic Bacteriology (2001), data in Tables 2-5 showed that the isolates were classified. In general to 3 genera including 5 species isolated from fertile soils and 7 genera including 8 species isolated from new reclaimed soils. Nostoc spp. strains were the most common filamentous heterocyst-forming ( 22 isolates). Two species of Nostoc including 11 isolates of $N$. muscorum (9 isolates from fertile soils and 2 isolates from reclaimed soils) and 11 isolates of $N$. calcicola ( 3 from fertile soils and 8 from reclaimed soils) were identified. Anabaena includes 10 isolates in three species (A. oryzae (6 isolates), A. circinalis ( 2 isolates from fertile soils) and A. oscillarioides (2 isolates from reclaimed soils) of Nodularia spumigena were represented in this work by two isolates from fertile soils. 


\begin{tabular}{|c|c|c|c|c|c|c|c|c|c|c|c|c|c|c|c|c|c|c|c|c|c|c|}
\hline \multicolumn{2}{|c|}{ Sheath } & & & & & & & & & & & + & & & & & & & & & & \\
\hline \multicolumn{2}{|c|}{ Baeocyste } & & & & & & & & & & & & & & & & & ' & ' & & & \\
\hline \multirow{3}{*}{ 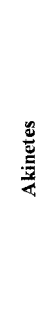 } & 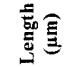 & $\begin{array}{l}\infty \\
r \\
r\end{array}$ & 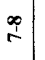 & $\infty$ & $\bar{n}$ & $\infty$ & 9 & $\hat{b}$ & $\begin{array}{l}\infty \\
0 \\
0\end{array}$ & 产 & $\$$ & $f^{\prime}$ & $f$ & $\infty$ & $\vec{b}$ & . & $\bar{n}$ & $\$$ & $\frac{\Xi}{b}$ & $\widetilde{b}$ & 象 & $\stackrel{\circ}{b}$ \\
\hline & 喜息 & 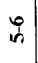 & 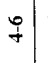 & ì & $q$ & 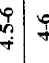 & $q$ & s & $\stackrel{0}{+}$ & 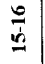 & $\hat{i n}$ & ? & if & $\tilde{n}$ & 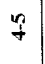 & $\bar{Z}$ & ? & f & $q$ & 7 & F & $\bar{n}$ \\
\hline & 旁 & 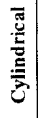 & 丞 & 烝 & 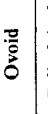 & 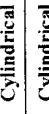 & 递 & 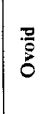 & 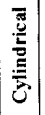 & 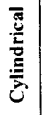 & 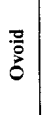 & 竎 & 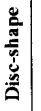 & 总 & 总 & 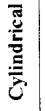 & 产 & 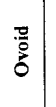 & $\begin{array}{l}\frac{\pi}{0} \\
\bar{o} \\
0\end{array}$ & 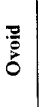 & & 总 \\
\hline \multicolumn{2}{|c|}{ 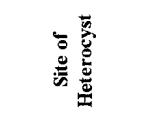 } & 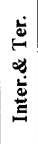 & | & . & | & 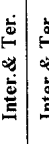 & 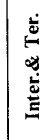 & 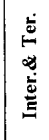 & 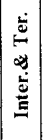 & 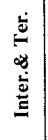 & 离 & | & 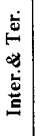 & 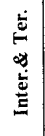 & 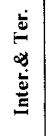 & 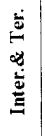 & 竧 & 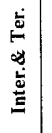 & 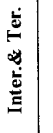 & | & & 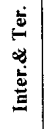 \\
\hline \multirow{3}{*}{ 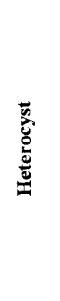 } & 熟重 & $\vec{m}$ & 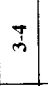 & \pm & 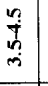 & $\Phi=$ & 贵 & $\begin{array}{l}n \\
0 \\
0 \\
0 \\
n \\
n\end{array}$ & \pm & $\stackrel{9}{1}$ & $q$ & $m$ & $\dot{m}$ & if & $q$ & $\stackrel{\infty}{2}$ & $q$ & $\begin{array}{c}0 \\
+\end{array}$ & if & 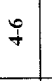 & is & $\begin{array}{l}0 \\
+\end{array}$ \\
\hline & 言金 & $\mid \begin{array}{l}n \\
0 \\
\vdots \\
i \\
i\end{array}$ & $\mid \begin{array}{l}n \\
3\end{array}$ & 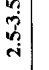 & 尊 & $\begin{array}{c}3 \\
8 \\
4\end{array}$ & $\stackrel{n}{3}$ & $\frac{n}{2}$ & $\mid \begin{array}{l}3 \\
\vec{b} \\
i\end{array}$ & î. & $\stackrel{n}{m}$ & \pm & $\dot{m}$ & $m$ & \pm & 资 & $\dot{m}$ & I & 吕 & मे & मे & $q$ \\
\hline & 耪 & 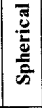 & 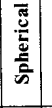 & 胥 & 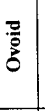 & $\begin{array}{ll}\frac{5}{5} \\
\frac{5}{2} \\
\bar{n}\end{array}$ & 咅 & 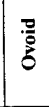 & 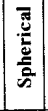 & 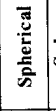 & 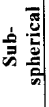 & 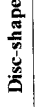 & & & 总 & & 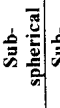 & & & & & \\
\hline \multirow{3}{*}{ 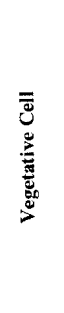 } & 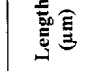 & $m$ & $\begin{array}{l}n \\
n \\
m\end{array}$ & : & $\stackrel{\square}{\ddagger}$ & $m$ & 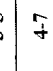 & F & 5 & $\begin{array}{l}\infty \\
0\end{array}$ & ir & मे & \pm & $\therefore$ & म & in & $I$ & $I$ & \pm & $\dot{m}$ & $\stackrel{\circ}{\dot{b}}$ & $\stackrel{0}{\ddagger}$ \\
\hline & 意衰 & $3:$ & 3 & $\stackrel{n}{i}$ & $\stackrel{m}{m}$ & $\stackrel{2}{2}$ & $\stackrel{n}{n}$ & 3 & 2 & $\mid \begin{array}{l}n \\
\vdots \\
6\end{array}$ & \pm & $\Phi$ & $\stackrel{n}{n}$ & \pm & 3 & $\dot{i}$ & $\stackrel{n}{i}$ & 2 & 3 & 2 & $\begin{array}{l}n \\
m \\
m\end{array}$ & $\mid \begin{array}{l}m \\
\tilde{3} \\
\dot{j} \\
i\end{array}$ \\
\hline & 品 & 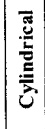 & & & & $\overline{\tilde{\pi}}$ & & & . & & & +1 & & & & & & & & & & $=\bar{s}$ \\
\hline \multicolumn{2}{|r|}{ |в.มиวэ) } & 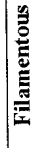 & $\mid$ & 资 & 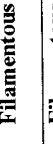 & 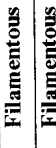 & & 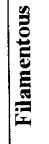 & 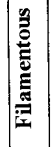 & 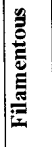 & 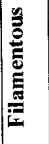 & 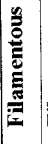 & 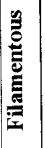 & 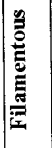 & 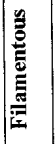 & 党 & 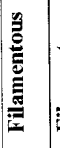 & & | & & & $\mid$ \\
\hline \multicolumn{2}{|r|}{ 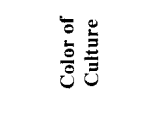 } & 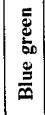 & 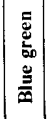 & 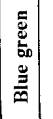 & 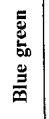 & $\mid$ & 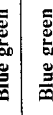 & 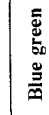 & 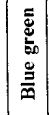 & 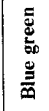 & 辩 & 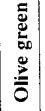 & 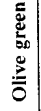 & 言 & 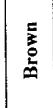 & 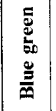 & $\frac{\bar{z}}{\dot{n}}$ & 童 & 言 & 熟 & 咅 & 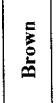 \\
\hline \multicolumn{2}{|c|}{ 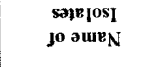 } & & $\bar{N}$ & ก & $\vec{k}$ & 9 & 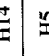 & $\tilde{n}$ & $\bar{a}$ & สี & $\frac{m}{2}$ & a & वे. & $\bar{\oplus}$ & $\Phi$ & $\dddot{m}$ & 苗 & \pm & స్త & 㽦 & $\overline{\vec{z}}$ & $\Xi$ \\
\hline
\end{tabular}


Isolation and Identification of Some Indigenous Cyanobacteria Isolates Based on

\begin{tabular}{|c|c|c|c|c|c|c|c|c|c|c|c|c|c|c|c|c|c|c|c|c|c|}
\hline \multicolumn{2}{|c|}{ чұвəчS } & & . & + & + & & & & & + & & . & & & & & & + & & + & + \\
\hline \multicolumn{2}{|c|}{ 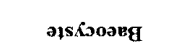 } & & & & & + & . & & & & & & & & & & & & + & & \\
\hline \multirow{3}{*}{ 离 } & 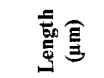 & $\infty$ & 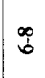 & & & & 条 & $\infty$ & $\infty$ & 号 & $\stackrel{i}{r}$ & 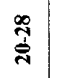 & $\tilde{A}$ & 吾 & in & ip & $\stackrel{\infty}{i}$ & 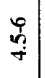 & & & \\
\hline & 意重 & $\stackrel{j}{q}$ & is & & & & $\stackrel{+}{+}$ & $\hat{n}$ & i⿱ & 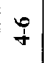 & के & $\overrightarrow{\bar{\phi}}$ & $\stackrel{9}{7}$ & if & in & if & $\begin{array}{l}n \\
\dot{q}\end{array}$ & 点 & & & \\
\hline & 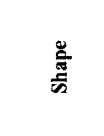 & 홓 & 豙 & & & & \begin{tabular}{|c|}
$\frac{5}{0}$ \\
$\overline{0}$
\end{tabular} & 竞 & : & 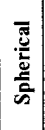 & $\frac{5}{0}$ & 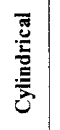 & 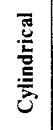 & 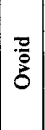 & 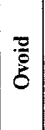 & $\frac{\pi}{c}$ & $\frac{\bar{z}}{b}$ & 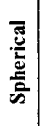 & & & \\
\hline \multicolumn{2}{|c|}{ 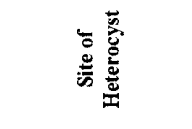 } & 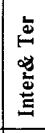 & 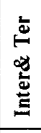 & & & & 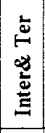 & 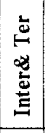 & 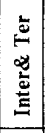 & 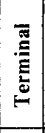 & 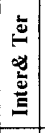 & 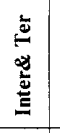 & 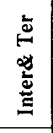 & 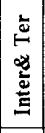 & 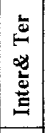 & 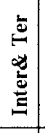 & 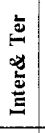 & . & & & \\
\hline \multirow{3}{*}{ 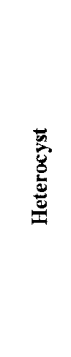 } & $\begin{array}{l}\text { 言 } \\
\text { 喜重 }\end{array}$ & if & $\stackrel{\circ}{\circ}$ & . & . & & b & $\stackrel{\circ}{\circ}$ & $\mid \begin{array}{l}n \\
0 \\
i \\
i n\end{array}$ & $\begin{array}{l}\infty \\
0 \\
0 \\
0\end{array}$ & $\left|\begin{array}{l}n \\
\vdots \\
0 \\
n \\
n\end{array}\right|$ & $\begin{array}{l}n \\
n \\
0 \\
0 \\
0\end{array}$ & $\hat{b}$ & 点 & $\left|\begin{array}{l}n \\
\vdots \\
0 \\
0 \\
m \\
m\end{array}\right|$ & $\frac{m}{j}$ & 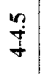 & $\hat{b}$ & 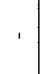 & . & . \\
\hline & 咅重 & $\stackrel{m}{m}$ & $\begin{array}{l}8 \\
8 \\
8 \\
\dot{y}\end{array}$ & . & . & & 品 & $\mid$\begin{tabular}{l}
0 \\
0 \\
$⿱ 亠 䒑$ \\
\hdashline \\
\end{tabular} & $\vec{m}$ & in & $m$ & $\overline{6}$ & $\infty$ & $\stackrel{m}{m}$ & 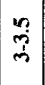 & $m$ & $m$ & $\stackrel{\circ}{\circ}$ & & . & . \\
\hline & 旁 & 冞 & 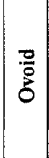 & ' & & & 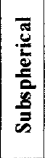 & ga & $\frac{7}{0}$ & 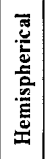 & 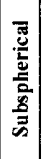 & 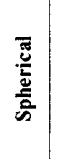 & 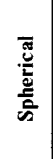 & 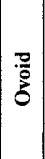 & \begin{tabular}{|l|} 
\\
\\
\\
$b$ \\
\end{tabular} & 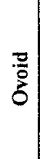 & 䇏 & 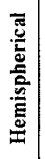 & & & \\
\hline \multirow{3}{*}{ 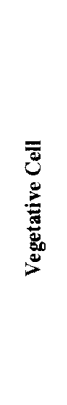 } & 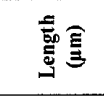 & 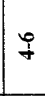 & $\stackrel{\circ}{\circ}$ & ơ & . & & in & is & 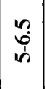 & 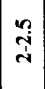 & $\sqrt{6}$ & $\stackrel{m}{9}$ & ஜ & $q$ & 7 & 7 & $q$ & $\begin{array}{l}0 \\
\overrightarrow{3} \\
\overrightarrow{3}\end{array}$ & & & 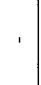 \\
\hline & 意要 & I & \pm & $\stackrel{n}{\sim}$ & 2 & $\bar{b}$ & 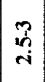 & 童 & $\begin{array}{l}2 \\
5 \\
4\end{array}$ & in & 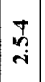 & $\begin{array}{l}n \\
6 \\
6 \\
q\end{array}$ & 号 & 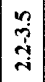 & $\mid \begin{array}{l}m \\
0 \\
m\end{array}$ & $\frac{n}{m}$ & $\frac{n}{m}$ & $\begin{array}{l}3 \\
2 \\
2\end{array}$ & $\stackrel{\dot{\sim}}{\mathrm{i}}$ & 3 & 2 \\
\hline & 惫 & 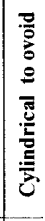 & 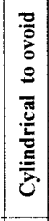 & 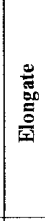 & 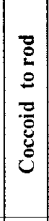 & 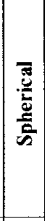 & 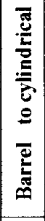 & 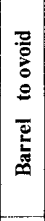 & 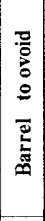 & 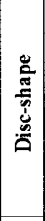 & 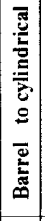 & & 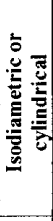 & 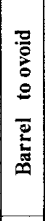 & 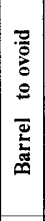 & 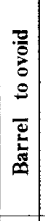 & 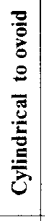 & 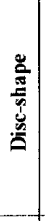 & 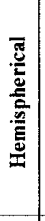 & 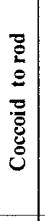 & 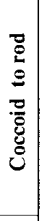 \\
\hline \multicolumn{2}{|r|}{ 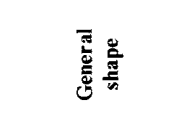 } & 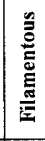 & 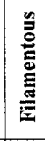 & 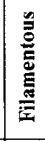 & 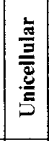 & 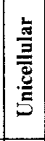 & 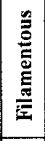 & 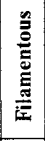 & 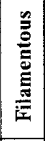 & 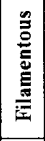 & 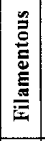 & 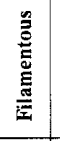 & 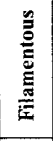 & 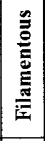 & 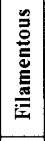 & 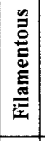 & 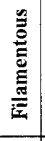 & 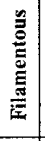 & 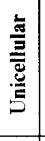 & 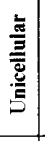 & 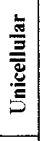 \\
\hline \multicolumn{2}{|c|}{ 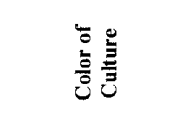 } & 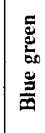 & 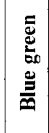 & 言 & 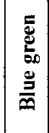 & 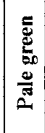 & 竞 & 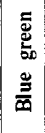 & 膀 & 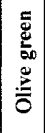 & 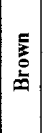 & 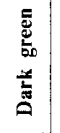 & 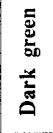 & 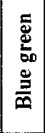 & 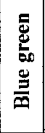 & $\mid$ & 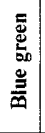 & 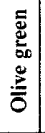 & 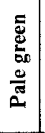 & 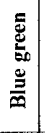 & 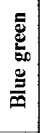 \\
\hline \multicolumn{2}{|c|}{ 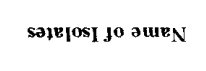 } & בี & 吾 & 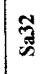 & $\frac{3}{3}$ & 虂 & $\overrightarrow{\underline{\mathrm{d}}}$ & 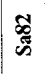 & 咅 & $\frac{\pi}{d}$ & $\mid \begin{array}{l}8 \\
\xi \\
\%\end{array}$ & $\frac{\pi}{\bar{H}}$ & $\frac{9}{\overline{8}}$ & $\frac{\pi}{\bar{z}}$ & 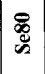 & 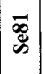 & 践 & $\overline{\bar{\omega}}$ & $\frac{O}{ \pm}$ & $\bar{z}$ & $\exists$ \\
\hline
\end{tabular}


Table (4): Physiological characters of cyanoprokaryotal isolates, isolated from fertile soils.

\begin{tabular}{|c|c|c|c|c|c|c|c|}
\hline \multirow{3}{*}{$\begin{array}{l}\frac{0}{\pi} \\
0 \\
0 \\
0 \\
0 \\
\dot{0} \\
\dot{z}\end{array}$} & \multirow[b]{3}{*}{$\begin{array}{c}\text { Total } \\
\text { protein } \\
(\mathrm{mg} / \mathrm{g})\end{array}$} & \multirow{3}{*}{ 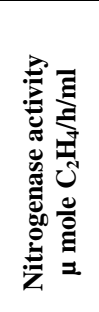 } & \multicolumn{5}{|c|}{ Pigments } \\
\hline & & & \multirow[b]{2}{*}{ 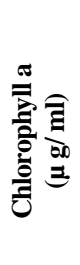 } & \multicolumn{3}{|c|}{ Phycocyanin } & \multirow[b]{2}{*}{ 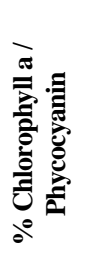 } \\
\hline & & & & 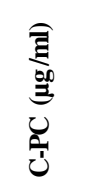 & 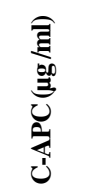 & 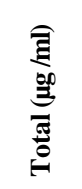 & \\
\hline Z1 & 270.3 & 16.049 & 9.91 & 17.90 & 35.70 & 53.60 & 18.49 \\
\hline Z11 & 295.0 & 9.351 & 1.69 & 2.90 & 6.70 & 9.60 & 17.60 \\
\hline Z12 & 308.7 & 12.764 & 1.53 & 2.50 & 7.00 & 9.50 & 16.11 \\
\hline $\mathbf{Z 5 1}$ & 221.4 & 6.429 & 3.63 & 7.00 & 9.40 & 16.40 & 22.13 \\
\hline H13 & 338.4 & 9.076 & 1.69 & 7.70 & 6.00 & 13.70 & 12.34 \\
\hline H14 & 254.7 & 7.801 & 2.83 & 9.30 & 7.80 & 17.10 & 16.55 \\
\hline H5 & 288.3 & 6.429 & 3.49 & 14.00 & 17.90 & 31.90 & 10.94 \\
\hline H52 & 265.2 & 5.000 & 1.35 & 4.70 & 2.30 & 7.00 & 19.29 \\
\hline D1 & 242.7 & 6.436 & 1.76 & 7.70 & 4.60 & 12.30 & 14.31 \\
\hline D2 & 283.5 & 5.502 & 1.34 & 7.50 & 5.10 & 12.60 & 10.63 \\
\hline D13 & 321.3 & 10.468 & 2.58 & 7.30 & 8.20 & 15.50 & 16.65 \\
\hline A2 & 244.5 & 1.301 & 4.52 & 4.10 & 37.40 & 41.50 & 10.89 \\
\hline A22 & 251.7 & 2.977 & 1.98 & 7.10 & 41.80 & 48.90 & 4.05 \\
\hline B1 & 234.6 & 3.051 & 9.68 & 15.20 & 20.00 & 35.20 & 27.50 \\
\hline B4 & 261.0 & 3.489 & 5.44 & 5.40 & 8.00 & 16.00 & 34.00 \\
\hline B5 & 303.9 & 4.735 & 5.35 & 7.60 & 14.10 & 21.70 & 24.65 \\
\hline E3 & 349.2 & 21.056 & 5.17 & 9.10 & 7.30 & 16.40 & 31.52 \\
\hline E4 & 261.0 & 3.489 & 5.44 & 8.70 & 7.30 & 16.00 & 34.00 \\
\hline E24 & 282.9 & 6.570 & 5.47 & 8.40 & 8.90 & 17.30 & 31.62 \\
\hline E41 & 273.9 & 4.270 & 6.67 & 6.80 & 8.20 & 15.00 & 44.47 \\
\hline M11 & 311.1 & 4.270 & 10.38 & 19.00 & 36.00 & 55.00 & 18.87 \\
\hline M12 & 335.7 & 9.316 & 9.61 & 14.40 & 33.60 & 48.00 & 20.02 \\
\hline
\end{tabular}

Based on morphological and physiological characterizations (Table 2 and 4) and according to Desikachary (1959), Rippka et al. (1979) and Bergey's Manual of Systematic Bacteriology (2001) the cyanoprokaryotal isolates were classified as follows:

$\begin{array}{ccclcr}\text { Z1 } & \text { Anabaena oryzae } & \text { D1 } & \text { Anabaena oryzae } & \text { E3 } & \text { Nostoc muscorum } \\ \text { Z11 } & \text { Anabaena oryzae } & \text { D2 } & \text { Anabaena circinalis } & \text { E4 } & \text { Nostoc muscorum } \\ \text { Z12 } & \text { Anabaena oryzae } & \text { D13 } & \text { Nostoc muscorum } & \text { E24 } & \text { Nostoc muscorum } \\ \text { Z51 } & \text { Nostoc calcicola } & \text { A2 } & \text { Nodularia spumigena } & \text { E41 } & \text { Nostoc muscorum } \\ \text { H13 } & \text { Anabaena oryzae } & \text { A22 } & \text { Nodularia spumigena } & \text { M11 } & \text { Nostoc muscorum } \\ \text { H14 } & \text { Anabaena oryzae } & \text { B1 } & \text { Nostoc muscorum } & \text { M12 } & \text { Nostoc muscorum } \\ \text { H5 } & \text { Nostoc calcicola } & \text { B4 } & \text { Nostoc muscorum } & & \\ \text { H52 } & \text { Nostoc calcicola } & \text { B5 } & \text { Anabaena circinalis } & & \end{array}$


Table (5) Physiological characters of cyanoprokaryotal isolates, isolated from new reclaimed soils

\begin{tabular}{|c|c|c|c|c|c|c|c|}
\hline \multirow{3}{*}{$\begin{array}{l}\stackrel{\mathscr{U}}{\pi} \\
\frac{\pi}{0} \\
\ddot{0} \\
\ddot{0} \\
\dot{0} \\
\dot{Z}\end{array}$} & \multirow[b]{3}{*}{$\begin{array}{c}\text { Total } \\
\text { protein } \\
(\mathrm{mg} / \mathrm{g})\end{array}$} & \multirow{3}{*}{ 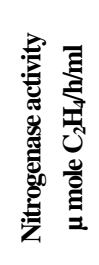 } & \multicolumn{5}{|c|}{ Pigments } \\
\hline & & & \multirow[b]{2}{*}{ 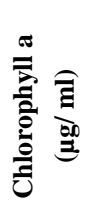 } & \multicolumn{3}{|c|}{ Phycocyanin } & \multirow{2}{*}{ 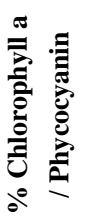 } \\
\hline & & & & 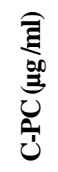 & 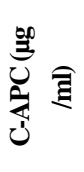 & 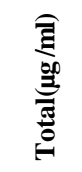 & \\
\hline Sa30 & 236.7 & 6.429 & 3.11 & 2.7 & 5.7 & 8.40 & 37.02 \\
\hline Sa31 & 321.3 & 13.210 & 9.22 & 8.8 & 27.7 & 36.50 & 25.26 \\
\hline Sa32 & 193.8 & 4.550 & 1.36 & 6.7 & 17.9 & 24.60 & 5.53 \\
\hline Sa33 & 222.0 & 0.067 & 1.37 & 4.0 & 4.5 & 8.50 & 16.12 \\
\hline Sa80 & 207.0 & 0.082 & 1.53 & 0.5 & 2.3 & 2.80 & 54.64 \\
\hline Sa81 & 313.5 & 14.370 & 3.46 & 3.4 & 6.7 & 10.10 & 34.26 \\
\hline Sa82 & 217.8 & 4.103 & 2.54 & 4.2 & 13.3 & 17.50 & 14.51 \\
\hline Sa83 & 297.3 & 9.316 & 7.07 & 3.9 & 18.0 & 21.90 & 32.28 \\
\hline Se12 & 245.1 & 8.384 & 4.86 & 3.4 & 5.4 & 8.80 & 55.23 \\
\hline Se100 & 307.2 & 15.010 & 1.99 & 4.8 & 4.4 & 9.20 & 21.63 \\
\hline Se112 & 259.5 & 4.664 & 4.98 & 4.6 & 5.3 & 9.90 & $\mathbf{5 0 . 3 0}$ \\
\hline Se113 & 221.7 & 2.454 & 3.60 & 3.7 & 3.2 & 6.90 & $\mathbf{5 2 . 1 7}$ \\
\hline Se114 & 260.7 & 5.101 & 1.17 & 2.1 & 1.9 & 4.00 & 29.25 \\
\hline Se80 & 302.1 & 10.450 & 0.73 & 1.7 & 3.2 & 4.90 & 14.90 \\
\hline Se81 & 187.5 & 3.057 & 1.19 & 2.2 & 6.8 & 9.00 & 13.22 \\
\hline Se82 & 274.8 & 6.837 & 0.76 & 5.6 & 2.4 & 8.00 & 9.50 \\
\hline Sh1 & 323.1 & 11.350 & 3.29 & 3.4 & 4.0 & 7.40 & 44.46 \\
\hline Sh10 & 206.4 & 0.052 & 1.92 & 1.0 & 2.1 & 3.10 & 61.94 \\
\hline Sh11 & 225.3 & 3.630 & 0.39 & 8.7 & 9.1 & 17.80 & 2.19 \\
\hline Sh111 & 240.3 & 0.740 & 0.76 & 3.6 & 14.8 & 18.40 & 4.13 \\
\hline
\end{tabular}

Based on morphological and physiological characterizations (Table 3 and 5) and according to Desikachary (1959), Rippka et al. (1979) and Bergey's Manual of Systematic Bacteriology (2001) the cyanoprokaryotal isolates were classified as follows:

$\begin{array}{ll}\text { Sa30 } & \text { Nostoc calcicola } \\ \text { Sa31 } & \text { N. calcicola. } \\ \text { Sa32 } & \text { Oscillatoria homogenea } \\ \text { Sa33 } & \text { Synechococcus sp } \\ \text { Sa80 } & \text { Xanococcus kerneri } \\ \text { Sa81 } & \text { N. muscorum } \\ \text { Sa82 } & \text { N. calcicola }\end{array}$

$\begin{array}{clcl}\text { Sa83 } & \text { N. calcicola } & \text { Se81 } & \text { N. calcicola } \\ \text { Se12 } & \text { Calothrix clavata } & \text { Se82 } & \text { N. calcicola } \\ \text { Se100 } & \text { N. muscorum } & \text { Sh1 } & \text { C. clavata } \\ \text { Se112 } & \text { Anabaena oscillarioides } & \text { Sh10 } & \text { Dermocarpa olivacea } \\ \text { Se113 } & \text { A. oscillarioides } & \text { Sh11 } & \text { Synechococcus sp } \\ \text { Se114 } & \text { N. calcicola } & \text { Sh111 } & \text { Synechococcus sp } \\ \text { Se80 } & \text { N. calcicola } & & \end{array}$


In addition Calothrix clavata (2 isolates), Synechococcus sp. (3 isolates) and one isolate of each Oscillatoria homogenea, Dermocarpa olivacea and Xanococcus kerneri were existed in the reclaimed soils. This indicates that Nostoc spp. and Anabaena spp. were spreading in fertile soils more than new reclaimed soils. The latter species were the most important cyanoprokaryota genera to fix atmospheric nitrogen in rice field.

Tables 2 and 3, show that the classification depended on the morphological characteristics has revealed that the cyanoprokaryota isolates namely D13, B1, B4, E3, E4, E24, E41, M11 and M12 (isolated from fertile soils) and Sa81, Se100 (isolated from reclaimed soils) were belong to Nostoc muscorum since they were characterized by filamentous, brown color culture. The shape of their vegetative cells ranged from barrel to cylindrical, and their diameter ranged from 2.0-4.0 $\mu \mathrm{m}$ in width and from 3.0-6.0 $\mu \mathrm{m}$ in length. Heterocysts were present in different isolates.

Their sites were found to be internal and terminal in the filament, their shapes ranged from spherical to ovoid, and their diameters ranged from 3.0-5.0 $\mu \mathrm{m}$ in width and from 4.0-6.0 $\mu \mathrm{m}$ in length. In the mentioned isolates, akinetes were present and their shapes were ovoid, and their diameters ranged from 4.0-7.0 $\mu \mathrm{m}$ in width and from 6.0-10.0 $\mu \mathrm{m}$ in length (Tables 2 and 3). On studying the physiological patterns of these isolates, data in Tables 4 and 5) revealed that their total protein content ranged from 261.0 to $349.2 \mathrm{mg} / \mathrm{g}$., nitrogenase activity ranged from 3.489 to $21.056 \mu$ mole $\mathrm{C}_{2} \mathrm{H}_{4} / \mathrm{h} / \mathrm{ml}$. and percentage of chlorophyll a / phycocyanin ranged from 16.65 to $44.47 \%$. the morphological characters and to some extent the physiological patterns of these isolates gave good reasons to classify them to Nostoc muscorum (Fig. 1a) according to Desikachary (1959) and Rippka et al. (1979).

In this respect, Komárek and Anagnostidis (1989) reported that in morphological characteristics are important for practical use and identification of natural population of cyanoprokaryota. The main phenotype character conforming to phylogenetic clusters (genera) inside of Nostocaceae is a metameric, symmetric or subsymmetric structure of trichomes resulting from the heterocyts and akinetes position (para- and apoheterocytic development of akinetes).

The ranking of strains for chlorophyll, PC, APC and PE content and also for their total phycobiliproteins and \% PBS out of total proteins can be very useful in selection of strains for their potential utilization as coloring agents, phycoflour probes or as additives in a range of cosmetic and pharmaceutical products (MacColl and Guard-Friar, 1987).

The isolates namely, Z51, H5 and H52 (isolated from fertile soils) and Sa30, Sa31, Sa82, Sa83, Se114, Se80, Se81 and Se82 (from new reclaimed soils) were belonging to Nostoc calcicola. They characterized by filamentous, color of culture ranged between blue green to brown. Vegetative cells shape ranged from 
barrel to ovoid, and their diameters ranged between 2.5-5.5 $\mu \mathrm{m}$ in width and 4.0$7.0 \mu \mathrm{m}$ in length. Heterocysts were present in all isolates. Their sites were found to be internal and terminal in the filament. Heterocysts were ovoid in shape, and their diameters ranged from 3.0-6.0 $\mu \mathrm{m}$ in width and from 3.5-6.5 $\mu \mathrm{m}$ in length. Akinetes were present and their shapes were ovoid and ranged from 4.0-6.0 $\mu \mathrm{m}$ in width and from 5.0-10.0 $\mu \mathrm{m}$ in length. After classification to the species level as $N$. calcicola, the physiological characteristics were also studied. The total protein content ranged from 187.5 to $321.3 \mathrm{mg} / \mathrm{g}$. Nitrogenase activity ranged from 3.056 to $13.210 \mu$ mole $\mathrm{C}_{2} \mathrm{H}_{4} / \mathrm{h} / \mathrm{ml}$. Percentage of chlorophyll a / phycocyanin ranged from 9.50 to $37.02 \%$. From the mentioned data and according to Rippka et al., (1979) and Bergey's Manual of Systematic Bacteriology (2001) these cyanoprokaryotal isolates were classified as Nostoc calcicola (Fig. 1b).

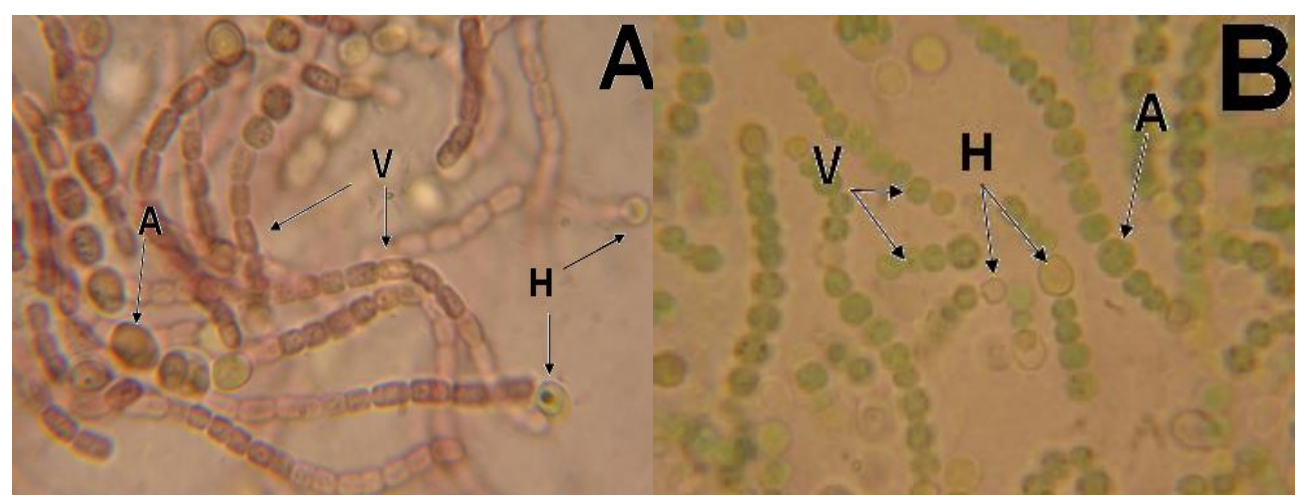

Figure (1): Light micrographs of (A) N. muscorum and (B) N. calcicola Where $A=$ Akinete, $H=$ Heterocyts and $V=$ Vegetative cell

Regarding the genus Anabaena, morphological classification indicated that the isolates namely, Z1, Z11, Z12, H13, H14 and D1 (isolated from fertile soils) were belong to $A$. oryzae (Tables 2 and 4), since they were characterized by filamentous shapes and bluish green in color. Shapes of vegetative cells were cylindrical and their diameters ranged from 1.5-3.5 $\mu \mathrm{m}$ in width and from 3.0-5.0 $\mu \mathrm{m}$ in length. Heterocysts were present and their sites were found to be internal and terminal in the filamentous. The heterocysts had a spherical shape, and diameter ranged from 2.5-3.5 $\mu \mathrm{m}$ in width and from 3.0-4.0 $\mu \mathrm{m}$ in length. Akinetes were present and were cylindrical in shape, their diameters ranged between $4.0-6.0 \mu \mathrm{m}$ in width and between $6.0-8.0 \mu \mathrm{m}$ in length. Physiological characteristics revealed that the total protein content ranged from 242.7 to 338.4 $\mathrm{mg} / \mathrm{g}$. Nitrogenase activity ranged from 6.436 to $16.049 \mu$ mole $\mathrm{C}_{2} \mathrm{H}_{4} / \mathrm{h} / \mathrm{ml}$. Percentage of chlorophyll $a$ / phycocyanin ranged from 12.34 to $18.49 \%$. Based 
on mentioned data and according to Desikachary (1959), Rippka et al. (1979), these cyanoprokaryotal isolates could be classified as Anabaena oryzae (fig 2a).

Traditionally, Anabaena species are identified according to their morphological characters such as morphology of filaments, vegetative cells, heterocysts, and akinetes (Li et al., 2000 and Rajaniemi et al., 2005).

Prasanna et al. (2006) stated that significant differences were observed among the Anabaena strains with regard to the shape and size of trichomes and individual cells within a filament, besides qualitative and quantitative aspects of phycobiliprotein accumulation and activities of enzymes involved in nitrogen metabolism.

Results in Tables (2 and 4) and Fig. (2) showed the morphological and physiological characteristics of the isolates namely, D2 and B5 (from fertile soils) which referred that to these isolates could be Anabaena circinalis. The isolates were well characterized by filamentous shapes, blue green in color. Vegetative cells ranged from spherical to ovoid, and their diameters ranged from 5.0-7.5 $\mu \mathrm{m}$ in width and from 5.0-8.0 $\mu \mathrm{m}$ in length. Heterocysts were present and their sites were found internal and terminal in the filament. Heterocysts shapes were spherical having diameters ranged from 5.5-9.5 $\mu \mathrm{m}$ in width and from 7.0-10.0 $\mu \mathrm{m}$ in length. Akinetes were present and cylindrical in shapes with diameters ranged between 14.0-17.0 $\mu \mathrm{m}$ in width and from 16.0-19.0 $\mu \mathrm{m}$ in length. Total protein content ranged from 283.5 to $303.9 \mathrm{mg} / \mathrm{g}$. Nitrogenase activity ranged from 4.735 to $5.502 \mu$ mole $\mathrm{C}_{2} \mathrm{H}_{4} / \mathrm{h} / \mathrm{ml}$. Percentage of chlorophyll a / phycocyanin ranged from 10.63 to $24.65 \%$. Based on the mentioned data these cyanoprokaryotal isolates were classified as Anabaena circinalis (Fig. 2b) (Desikachary, 1959 and Rippka et al., 1979).

Li et al. (2000) reported that Anabaena circinalis characterized by vegetative cells with gas vesicles, spherical and 5.3-11.7 $\mu \mathrm{m}$ in diameter. Heterocyts are spherical and 10.7-12.9 $\mu \mathrm{m}$ in diameter. Akinetes ellipsoidal with conical ends, 13.0-17.5 $\mu \mathrm{m}$ in diameter, 23.4-30.7 $\mu \mathrm{m}$ in length, distant from heterocytes.

In the same Tables (2 and 3) the cyanoprokaryotal isolates namely, Se112 and Se113 (isolated from new reclaimed soils) could be classified as Anabaena oscillarioides which were well characterized by filamentous and dark green in color. Their vegetative cells ranged from isodiametric to cylindrical shapes, and their diameters ranged from 4.5-6.0 $\mu \mathrm{m}$ in width and from 4.0-6.5 $\mu \mathrm{m}$ in length. The heterocysts were present and their sites were found internal and terminal in the filament. Their shapes were spherical, having diameters ranged from 6.0-8.0 $\mu \mathrm{m}$ in width and 6.0-9.0 $\mu \mathrm{m}$ in length. Akinetes were present with cylindrical shapes. Their diameter ranged between 7.0-11.0 $\mu \mathrm{m}$ in width and between 20.0$28.0 \mu \mathrm{m}$ in length. The same features were also used for morphological classification of Anabaena species by Rajaniemi et al. (2005). The physiological 
study demonstrated that the cells contains total protein content ranged from 221.7 to $259.5 \mathrm{mg} / \mathrm{g}$., nitrogenase activity ranged from 2.454 to $4.664 \mu$ mole $\mathrm{C}_{2} \mathrm{H}_{4} / \mathrm{h} / \mathrm{ml}$. and percentage of chlorophyll $a$ / phycocyanin ranged from 50.30 to $52.17 \%$. The observation of cyanoprokaryota Anabaena oscillarioides under the microscope is shown in Fig. (2c).

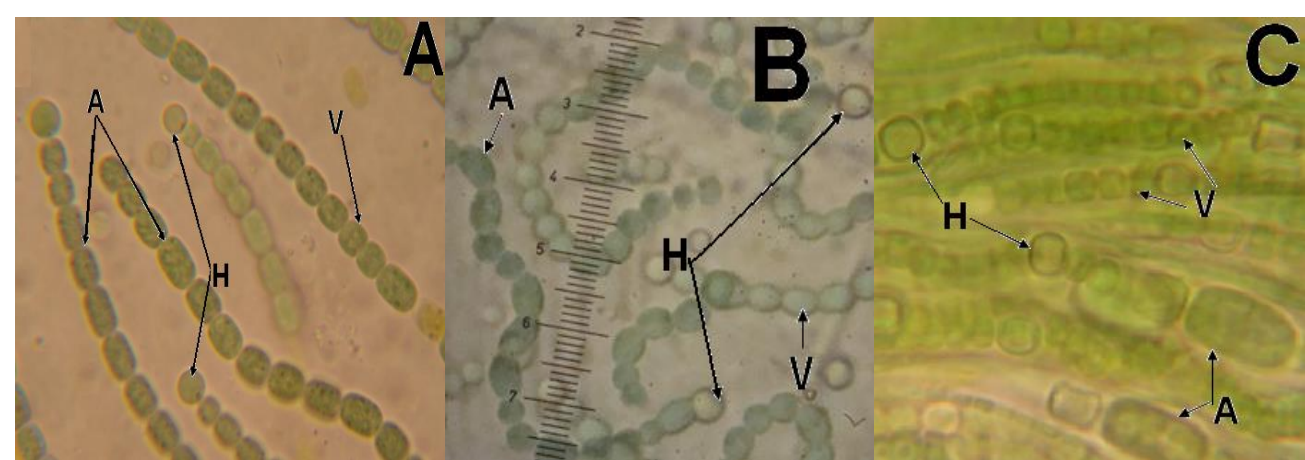

Figure (2): Light micrographs of (A) Anabaena oryzae, (B) A. circinalis and (C) A. oscillarioides. Where $A=$ Akinete, $\mathrm{H}=$ Heterocyts and $\mathrm{V}=$ Vegetative cell

The isolates namely, A2 and A22 (from fertile soils) could be classified as Nodularia spumigena according to the morphological characters presented in Table ( 2 and 4$)$. These isolates were characterized by being filamentous and olive green in color. Vegetative cells were disk shape, and their diameters ranged from $3.0-4.0 \mu \mathrm{m}$ in width and from $3.0-4.0 \mu \mathrm{m}$ in length. Heterocysts were present and their sites were found internal and terminal in the filament. Their shapes were a disk-shape, and their diameters ranged from 3.0 -4.0 $\mu \mathrm{m}$ in width and from 3.0 $5.0 \mu \mathrm{m}$ in length. Akinetes were present and their shapes were disk-shape and diameters ranged from 4.0 $-5.0 \mu \mathrm{m}$ in width and from $4.0-5.0 \mu \mathrm{m}$ in length. Sheaths were present and total protein content ranged from 244.5 to $251.7 \mathrm{mg} / \mathrm{g}$. Nitrogenase activity ranged from 1.301 to $2.977 \mu$ mole $\mathrm{C}_{2} \mathrm{H}_{4} / \mathrm{h} / \mathrm{ml}$. Percentage of chlorophyll a / phycocyanin ranged from 4.05 to $10.89 \%$. Therefore, these cyanoprokaryotal isolates were classified as Nodularia spumigena (Fig.3a). in this aspect Komárek and Anagnostidis (1989) demonstrated that heterocyst differentiation in Nostoc, Anabaena, Cylindrospermum and Nodularia, always occurs in an intercalary position, but in Anabaena cylindrica, conical end cells sometimes give rise to similarly shaped heterocysts and may become terminal after trichome breakage.

The isolates namely, Se12 and Sh1 (Calothrix clavata) were well characterized by filamentous culture and olive green in color. The vegetative cells were disk shape, and their diameters ranged from 2.0 -3.0 $\mu \mathrm{m}$ in width and from $2.0-3.5 \mu \mathrm{m}$ in length (Table 3). In these isolates heterocysts were present and 
their sites were found terminal in the filament only. Their shape is a hemispherical and diameter ranged from 5.0 -7.0 $\mu \mathrm{m}$ in width and from 6.0 $-8.0 \mu \mathrm{m}$ in length. Akinetes were also present and their shapes were spherical having diameters ranged from $4.0-6.0 \mu \mathrm{m}$ in width and from $4.5-6.0 \mu \mathrm{m}$ in length. Sheaths were present in these isolates. The physiological patterns revealed that the total protein content ranged from 245.1 to $323.1 \mathrm{mg} / \mathrm{g}$., nitrogenase activity ranged from 8.384 to $11.350 \mu$ mole $\mathrm{C}_{2} \mathrm{H}_{4} / \mathrm{h} / \mathrm{ml}$. and percentage of chlorophyll a / phycocyanin ranged from 44.46 to $55.23 \%$ (Table 5). On these grounds, these cyanoprokaryotal isolates were classified as Calothrix clavata (Fig. 3b).

Bohuslav (2007) reported that the Calothrix can be recognized by trichomes heteropolar, at the basis with hemispherical terminal heterocyst, 7.5-10 $\mu \mathrm{m}$ in diameter. Cells at basis narrowelliptic or narrow-cylindrical creating spore, often with clear constrictions by cross walls, $5.5-12.5 \mu \mathrm{m}$ in diameter. Cells in the middle zone of trichome wider than longer or isodiametricm $2.5-3.5 \mu \mathrm{m}$ in diameter. Initial stages 6-10-celled, heteropolar, later creating specific basal wideness and apical hair.

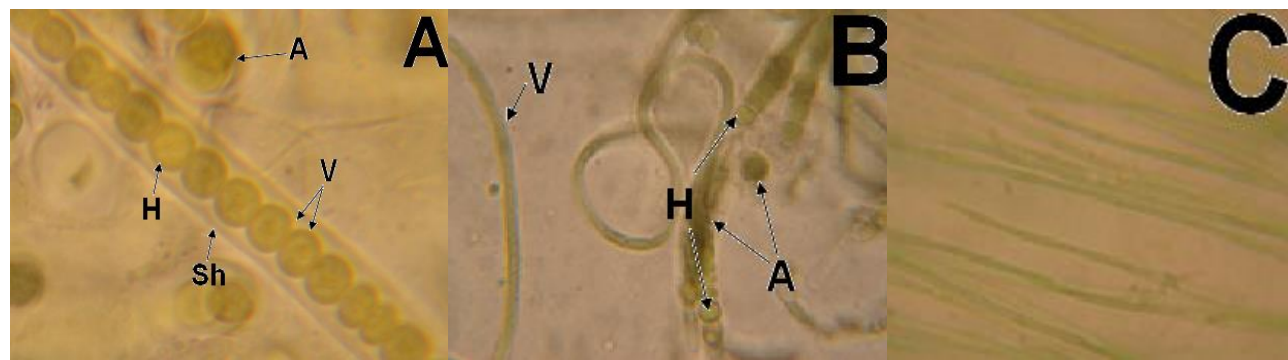

Figure (3): Light micrographs of (A) Nodularia spumigena, (B) Calothrix clavata and

(C) Oscillatoria homogenea. Where A= Akinete, $\mathrm{H}=$ Heterocyts, $\mathrm{V}=$ Vegetative cell and $\mathrm{Sh}=$ Sheath

Oscillatoria homogenea presented by isolate Sa32 (Table 3) demonstrated that this isolate was characterized by filamentous culture and green in color. The vegetative cells were elongate, and their diameters ranged from $2.0-3.5 \mu \mathrm{m}$ in width and from $4.0-6.0 \mu \mathrm{m}$ in length. Heterocysts, akinetes and sheaths were absent. These cells contains total protein amounted $193.8 \mathrm{mg} / \mathrm{g}$., nitrogenase activity $4.550 \mu$ mole $\mathrm{C}_{2} \mathrm{H}_{4} / \mathrm{h} / \mathrm{ml}$., and percentage of chlorophyll $a$ /phycocyanin $5.53 \%$ (Table 5). This isolate was, therefore, classified as Oscillatoria homogenea (Fig. 3c).

Pinevich (2008) reported that Oscillatoria are trichome cyanoprokaryota having no ability to form akinetes and heterocysts. Oscillatoria have no true branching, though in some cases, when the sheath was formed, false branching was observed. 
The isolates, Sa33, Sh11 and Sh111 classified as Synechococcus sp. were characterized by being unicellular cells (Table 3 ) reproduction by binary fission, color of culture was blue green. Their vegetative cells were coccoid to rod, and their diameters ranged from $1.0-3.0 \mu \mathrm{m}$. Sheaths were present. The physiological studies showed that the total protein content in the cells ranged from 222.0 to $240.3 \mathrm{mg} / \mathrm{g}$., nitrogenase activity from 0.067 to $3.630 \mu$ mole $\mathrm{C}_{2} \mathrm{H}_{4} / \mathrm{h} / \mathrm{ml}$., and percentage of chlorophyll $a$ / phycocyanin ranged from 2.19 to $16.12 \%$ (Table 5). These isolates were therefore, classified as Synechococcus sp. according to Rippka et al. (1979).

Synechococcus sp. is small unicellular cyanoprokaryota with ovoid to cylindrical cells that reproduce by binary traverse fission in a single plane. Its size varies from $0.8 \mu \mathrm{m}$ to $2.5 \mu \mathrm{m}$ (Rippka et al., 1979).

Komárek (2010a) stated that Synechococcus are unicellular cyanoprokaryota with more or less rod-like cells are usually classified into this genus. They are commonly distributed, easily grown in cultures and many of strains have been isolated.

Results in Tables (3 and 5) and Fig. (4) show also that the isolate Sh10 (Dermocarpa olivacea) was characterized by being unicellular cyanoprokaryota, reproduction by multiple fission, pale green in color. The vegetative cells were hehispherical, and their diameters ranged from 2.0-4.0 $\mu \mathrm{m}$. Baeocyste was present. On studying the physiological patterns of this isolate (Table 5) revealed that the total protein content in microbial mass was $206.4 \mathrm{mg} / \mathrm{g}$., and nitrogenase activity showed $0.052 \mu$ mole $\mathrm{C}_{2} \mathrm{H}_{4} / \mathrm{h} / \mathrm{ml}$. and percentage of chlorophyll a / phycocyanin was $61.94 \%$. The isolate was, therefore, classified as Dermocarpa olivacea according to the mentioned references.

The isolate Sa80 classified as Xanococcus kerneri was characterized by being unicellular simple (Table 3) reproduction by multiple fission. The observed color of culture was pale green. Its vegetative cells were spherical, and their diameters ranged from $6.0-11.0 \mu \mathrm{m}$. Baeocyste was present. In this isolate total protein value was $207.0 \mathrm{mg} / \mathrm{g}$., and nitrogenase activity gave $0.082 \mu$ mole $\mathrm{C}_{2} \mathrm{H}_{4} / \mathrm{h} / \mathrm{ml}$. The chlorophyll $a /$ phycocyanin amounted $54.60 \%$ (Table 5). Based on the mentioned data and according to Desikachary (1959) and Rippka et al. (1979), this isolate was classified as Xanococcus kerneri.

Zhaxybayeva et al. (2008) reported that single-celled ranged in size from about $0.6 \mu \mathrm{m}$ to well over $30 \mu \mathrm{m}$ in their largest dimension. Although, most species exceed $2 \mu \mathrm{m}$, the upper limit of organisms recognized as picoplankton, it seems likely that, based on world-wide biomass, the majority are below this limit.

In general, the identification indicated that Nostoc strains were the most common filamentous heterocyst -forming cyanoprokaryota the latter includes 22 strains of Nostoc (52.4\% of all strains) belongs to 2 species. Anabaena spp. was recorded as a second rank (23.8\% of all species). 


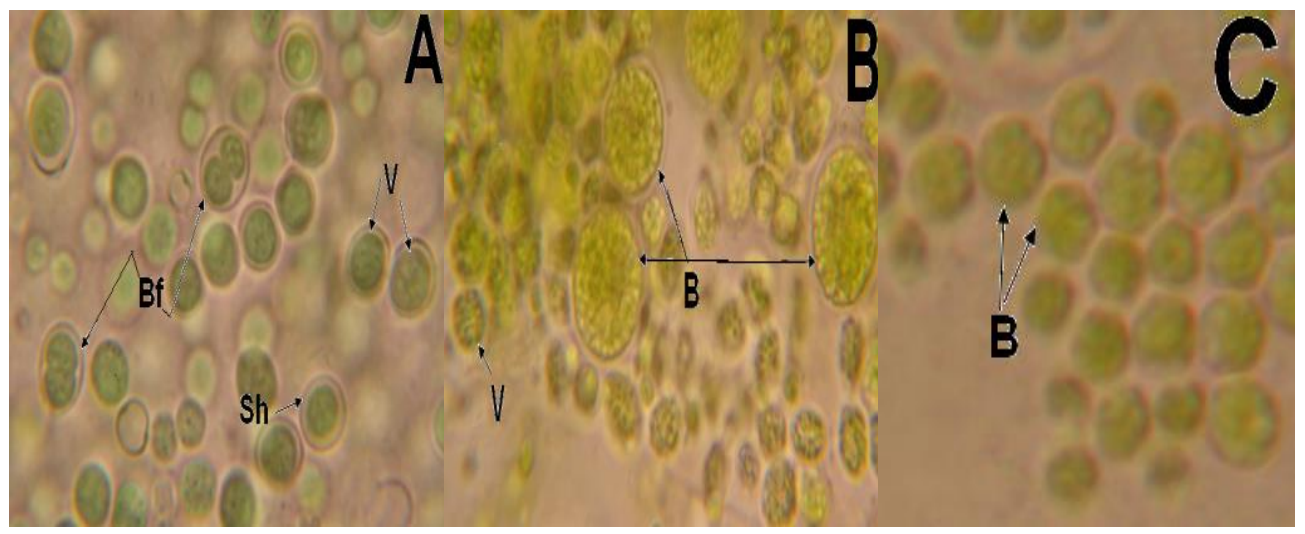

Figure (4): Light micrographs of (A) Synechococcus sp, (B) Dermocarpa olivacea and (C) Xanococcus kerneri. Where $\mathrm{Bf}=$ Binary fission, $\mathrm{B}=\mathrm{Baeocyste}, \mathrm{V}=$ Vegetative cell and $\mathrm{Sh}=$ Sheath

Regarding the distribution of cyanoprokaryotal communities in Sharkia governorate soils, a few isolates of unicellular species were found with the majority being filamentous with heterocysts. In a field experiment, Yanni (1991) studied the profiles of cyanoprokaryotal genera dominant in rice field by plating and microscopic methods. The population profile comprised $64 \%$ Nostoc, 24\% Anabaena and 8\% Calothrix. Aulosira, Nodularia, and Tolypothrix represented less than $1 \%$ of the total heterocystous cyanoprokaryota.

The dominance of the Nostoc genus has also been reported in other terrestrial habitats (Olson et al., 1998). The predominance of Nostoc in certain habitats has been attributed to its ability to remain desiccated for months to years, then recover metabolic activity within hours to days after rehydration with water, its ability to screen damaging UV light in terrestrial and shallow benthic habitats, its resistance to grazing probably due to production of a large amount of sheath material and the formation of colonies that are too large for many algivores to consume (Dodds et al., 1995).

Stulp (1982) concluded from studies on morphological variability of Anabaena strains that traditionally accepted criteria in the taxonomy of the genus Anabaena are stable over a wide range of environmental conditions and hence serve their utility for taxonomy.

The present investigation clearly brought out the diversity existing among the cyanoprokaryotal isolates analyzed in terms of various morphological and physiological attributes. Molecular polymorphisms further aided in effective differentiation of the selected strains, besides revealing their genetic relationships. A combination of attributes, including morphological and physiological 
characteristics could provide useful taxonomic criteria for distinguishing isolates belonging to the cyanoprokaryotal group, when analyzed under a uniform set of conditions (Prasanna et al., 2006). Such attributes should also be included in the descriptions of the genus/species in taxonomic treatises, for providing a more comprehensive characterization of the members placed under this group. This would facilitate not only selection of particular strain(s) for in-depth analyses of various metabolic processes but also for their effective utilization for diverse purposes (Shalini et al., 2008).

\section{References}

Bennett, A. and Bogorad, L. (1973). Complementary chromatic adaptation in filamentous blue-green algae. J. Cell. Biol., 58: 419 - 435.

Bergey's Manual of Systematic Bacteriology $\mathbf{2}^{\text {nd }}(2001)$. Rippka, R., R.W. Castentholtz, I. Iteman and M. Herdman (2001). Form-genus I. Anabaena Bory. In: Boone, D.R. and W.R. Castenholz, (Eds.). Springer, Berlin, pp. $566-568$.

Bohuslav, U. (2007). Morphological characterization of three subaerial Calothrix species (Nostocales, cyanobacteria). Fottea, 7(1): 33-38.

Bremner, J. M., and Mulvaney, C. S. (1982). Nitrogen - Total. In: Methods of Soil Analysis (A. L. Page et al., ed.). Agronomy Monograph 9, Part 2, $2^{\text {nd }}$ ed. American Society of Agronomy. Madison. WI. pp. 595-624.

Cardozo, K. H. M.; Guaratini, T.; Barros, M. P.; Falcão, V. R.; Tonon, A. P.; Lopes, N. P.; Campos, S.; Torres, M. A.; Souza, A. O.; Colepicolo, P. and Pinto, E. (2007). Metabolites from algae with economical impact. Comp. Biochem. Physiol. C. Toxicol. Pharmacol., 146: 60-78.

Castenholz, R. W. and Waterbury, J. B. (1989). Cyanoprokaryota. In: Staley, J. T., M. P. Bryant, N. Pfennig \& J. G. Holt (eds), Bergey's Manual of Systematic Bacteriology. Williams \& Wilkins, Baltimore. Vol., 3: 17101727.

Desikachary, T.V. (1959). Cyanophyta. ICAR Monograph on Algae. ICAR, New Delhi, India.

Desikachary, T.V. (1970). Taxonomy of blue-green algae: problems and prospects. Schweiz. Z. Hydrobiol., 32: 490-494.

Dodds, W.K.; Gudder, D.A. and Mollenhauer, D. (1995). The ecology of Nostoc. J. Phycol., 31: 2-18.

Ferris, M. J. and Hirsch, C. F. (1991). Method for isolation and purification of cyanobacteria. Appl. Environ. Microbiol., 57(5): 1448-1452.

Garcia-Pichel, F.; Sherry, N. D. and Castenholz, R. W. (1992). Evidence for an ultraviolet sunscreen role of the extracellular pigment scytonemin in the terrestrial cyanobacterium Chlorogloeopsis sp. Photochem. Photobiol., 56: 17-23. 
Häder, D. P.; Kumar, H. D.; Smith, R. C. and Worrest, R. C. (2007). Effects of solar UV radiation on aquatic ecosystems and interactions with climate change. Photochem. Photobiol. Sci., 6: 267-285.

Hardy, R. W. F.; Burnse, B. C. and Holstem, R. O. (1973). Application of the acetylene ethylene assay for the measuremenet of nitrogen fixation. Soil boil. Biochem., 5: 47-81.

Haselkorn, R. and Buikema, W.J. (1992). Nitrogen fixation by cyanoprokaryota. In: Stacey, G., Burris, R.H., Evans, H.J. (Eds.), Biological Nitrogen Fixation. Chapman \& Hall, New York, pp. 166-190.

Hindak, F. (2000). Morphological variation of four planktic nostocalean cyanophytes - members of the genus Aphanizomenon or Anabaena. Hydrobiologia, 438: 107-116.

Komàrek, J. (2010a). Recent changes (2008) in cyanobacteria taxonomy based on a combination of molecular background with phenotype and ecological consequences (genus and species concept). Hydrobiologia, 639:245-259.

Komàrek, J. (2010b). Modern taxonomic revision of planktic nostocacean cyanobacteria: a short review of genera. Hydrobiologia, 639:231-243.

Komàrek, J. and Anagnostidis, K. (1989). Modern approach to the classification system of cyanophytes, 4-Nostocales. Arch. Hydrobiol. Suppl. 823, Algolog. Stud., 56: 247-345.

Li, R.; Watanabe, M. and Watanabe, M. M. (2000). Taxonomic studies of planktic species of Anabaena based on morphological characteristics in cultured strains. Hydrobiologia, 438: 117-138.

MacColl, R. and Guard-Friar, D. (1987). Phycobiliproteins. CRC Press, Boca Raton, FL.

Meeks, J.C. and Castenholz, R.W. (1971). Growth and photosynthesis in an extreme thermophile, Synechococcus lividus (Cyanophyta). Arch. Microbiol., 78: 25-41.

Olson, J. B.; Steppe, T. F.; Litaker, R. W. and Paerl, H. W. (1998). $\mathrm{N}_{2}$-fixing microbial consortia associated with the ice cover of lake Bonney, Antarctica. Microb. Ecol., 36: 231-238.

Pinevich, A. V. (2008). Paradoxes of Biodiversity, Phylogeny, and Taxonomy of cyanobacteria. Moscow University Biological Sciences Bulletin, 63 (1): 2124.

Prasanna, R.; Kumar, R.; Sood, A.; Prasanna, B. M. and Singh, P. K. (2006). Morphological, physiochemical and molecular characterization of Anabaena strains. Microbiological Research, 161: 187-202.

Rajaniemi, P.; Hrouzek, P.; Kastovska, K.; Willame, R.; Rantala, A.; Hoffmann, L.; Komarek, J. and Sivonen, K. (2005). Phylogenetic and morphological evaluation of the genera Anabaena, Aphanizomenon, Trichormus and Nostoc (Nostocales, cyanobacteria). International Journal of Systematic and Evolutionary Microbiology, 55: 11-26.

Egyptian J. of Phycol. Vol. 12, $2011 \quad$ - 30 - 
Rippka, R.; Deruelles, J.; Waterbury, J.B.; Herdman, M. and Stanier, R.Y. (1979). Generic assignments strain histories and properties of pure cultures of cyanobacteria. J. Gen. Microbiol., 111: 1- 61.

Roger, P. A. and Kulasooriya, S. A. (1980). Blue green algae and rice. Int. Rice Res. Inst., los Banos, Philippines.

Shalini, A. E.; Dhar, D. W. and Gupta, R. K. (2008). Phylogenetic analysis of cyanobacteria strains of genus-Calothrix by single and multiplex randomly amplified polymorphic DNA-PCR. World J. Microbiol. Biotechnol., 24:927-935.

Singh, N. K.; Parmar, A. and Madamwar, D. (2009). Optimization of medium components for increased production of C-phycocyanin from Phormidium ceylanicum and its purification by single step process. Bioresource Technology, 100: 1663-1669.

Stainer, R. Y.; Kunisawa, R.; Mandel, M. and Cohen-Bazire, G. (1971). Purification and properties of unicellular blue-green algae (order Chroococales). Bact. Rev., 35: 171-205.

Stulp, B. K. (1982). Morphological variability of Anabaena strains (Cyanophyceae) under different culture conditions. Arch. Hyrdrobiol., 63: 165-176.

Vaishampayan, A.; Sinha, R. P.; Häder, D. P.; Dey, T.; Gupta, A. K.; Bhan, U. and Rao, A. L. (2001). Cyanoprokaryotal biofertilizers in rice agriculture. Bot. Rev., 67: 453- 516.

Yanni, Y. G. (1991). Potential of endogenous cyanoprokaryota to contribute to rice performance under different schedules of nitrogen application. World J. Microbiol. and Biotech., 7: 48-52.

Zhaxybayeva, O.; Doolittle, W. F.; Papke, R. T. and Gogarten, J. P. (2008). Intertwined evolutionary histories of marine Synechococcus and Prochlorococcus marinus genome. Biol. Evol., 1:325-339. 


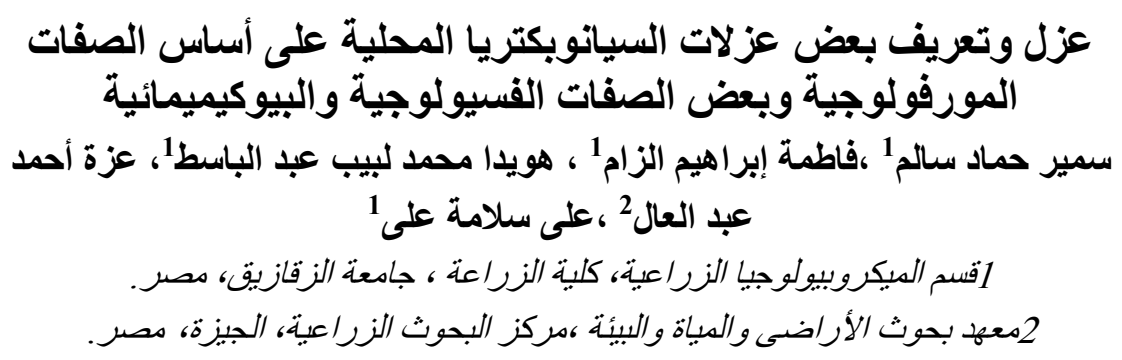

تم عزل 42 سلالة من السيانوبكتبريا من 10 أماكن مختلفة على إمتداد محافظة الشرقية، مصر الإنا

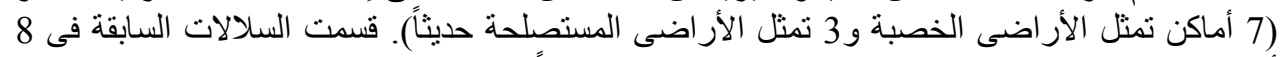

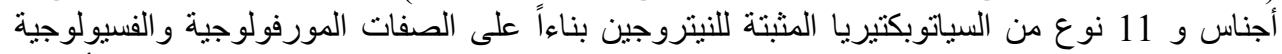

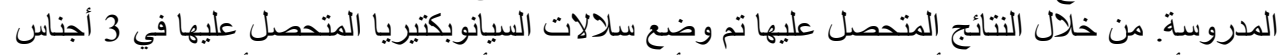

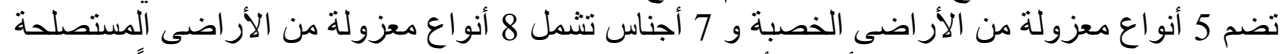

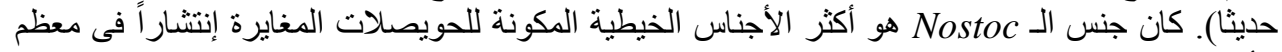

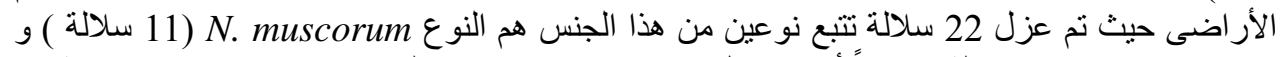

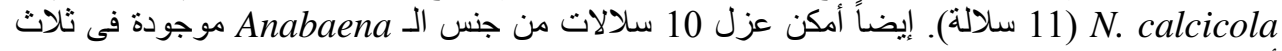

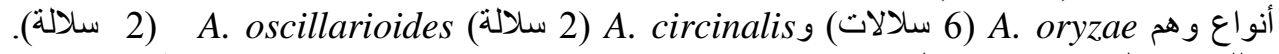

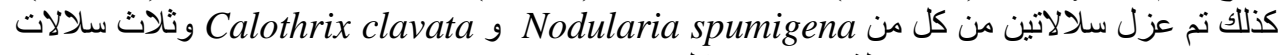

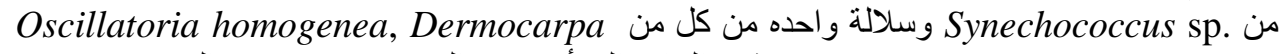
Anabaena olivacea , Xanococcus kerneri

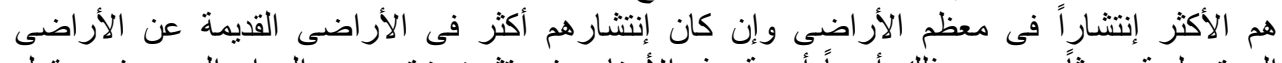

المستصلحة حديثاً ويوضح ذلك أيضاً أهمية هذه الأجناس فى تثبيث نيتروجين الهواء الأنى الجوى فى حقول الأرى

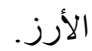

